\title{
Ulrich Ammon \\ Deutsch als plurizentrische Sprache und der Intertourismus zwischen den Zentren
}

\begin{abstract}
After a short historical summary which shows how today's political and geographic distribution of the German language has evolved, the concept of a pluricentric language is developed, with German being a case in point. Following this, the various - seven to ten - "centers" of the language are outlined, illustrated by maps, with their prominent linguistic, demographic and political features, as a possible approach toward a comprehensive typology of the centers of pluricentric languages. It is argued that each center's specific standard variants, which make it an (autonomous) center of the language, are fully valid and correct within their respective political realm. Furthermore, the Variantenwörterbuch des Deutschen is presented as the first comprehensive dictionary of a pluricentric language, containing the entire pluricentric variation of German. It is based on a newly conceived dictionary structure that provides easy access to any pluricentrally relevant linguistic feature. The central topic of the contribution is, however, the level of attraction of the various centers of a pluricentric language as tourist destinations for the inhabitants of the other centers of the same language. The study includes hints at possible methods of exploitation by the tourist industry - a topic also worthy of study for other pluricentric languages.
\end{abstract}

Keywords: 'pluricentric language', German language, 'center of a language', typology of the centers of pluricentric languages, comprehensive dictionaries of pluricentric languages, centers of pluricentric languages as tourist destinations

\section{Themenspezifizierung}

Deutsch gehört zu den wichtigen Tourismussprachen in allen europäischen, besonders den mediterranen Ländern. Allerdings rangiert es insgesamt weit hinter Englisch. Vermutlich liegt Deutsch als Tourismussprache aufs Ganze gesehen auch etwas hinter Französisch, aber vermutlich vor Italienisch, Spanisch, Russisch oder gar Portugiesisch - bei geographisch unterschiedlichen Verteilungen. Diese Aussage gilt für die internationalen europäischen Sprachen, also diejenigen, die ziemlich weltweit als Fremdsprachen gelernt werden (Definition in Ammon 2015: 18-33), und betrifft vor allem den Ausreisetourismus aus den Ländern oder Regionen dieser Sprachen, wo sie staatliche Amtssprache oder vorherrschende Muttersprache sind. Abgeschwächt gilt

Ulrich Ammon, Universität Duisburg-Essen, Deutschland, ulrich.ammon@uni-due.de 
sie vermutlich auch über Europa hinaus. Der Einreisetourismus in die deutschsprachigen Länder und Regionen hat ebenfalls eine beachtliche Größenordnung (zum Einreisetourismus Ammon 2015: 836-844, zum Ausreisetourismus ebd.: 844-864).

Statt der notgedrungen sehr pauschalen oder selektiven, allgemeinen Beschreibung des mit der deutschen Sprache zusammenhängenden Tourismus beschränke ich mich hier auf ein spezielleres Thema, zu dem die Betrachtung des Gebiets anregt, in dem Deutsch staatliche Amtssprache ist. Dieses ist gänzlich beschränkt auf Mitteleuropa - im Gegensatz zu anderen internationalen Sprachen wie Englisch, Französisch, Spanisch oder Portugiesisch. Hinsichtlich dieser Beschränkung gleicht Deutsch dem Italienischen. Der entscheidende Grund ist in beiden Fällen, dass auf dem ursprünglichen Gebiet dieser Sprachen lange Zeit kein mächtiger Staat entwikkelt wurde, der sich an der gewissermaßen klassischen Kolonialpolitik der europäischen Staaten beteiligen konnte - was ich hier nur zur Erklärung anführe, aber nicht beklage. Im deutschen Sprachgebiet war die mangelnde Staatsbildung bedingt durch die schwache Zentralgewalt im Heiligen Römischen Reich (Deutscher Nation) (10. Jahrhundert - 1806) und dem darin möglichen politischen Territorialismus sowie die konfessionelle Zersplitterung durch Reformation und Gegenreformation, die nach dem 30-Jährigen Krieg (1618-1648) das deutsche Sprachgebiet in rund 300 Kleinstaaten zerstückelte.

Ein größerer Nationalstaat und eine - kurzzeitige - Kolonialpolitik entstanden erst in der zweiten Hälfte des 19. Jahrhunderts. Diese neuere Geschichte verursachte eine zweite Besonderheit des heutigen deutschen Sprachgebiets (außer seiner amtssprachlichen Einschränkung auf Mitteleuropa), nämlich die Aufteilung auf viele Staaten. Schon als staatliche Amtssprache erstreckt sich Deutsch in 7 verschiedene Staaten hinein (Deutschland, Österreich, Schweiz, Liechtenstein, Luxemburg, Belgien und Italien), und hinzukommen noch deutschsprachige Minderheiten in mindesten 5 weiteren Staaten (Frankreich, Dänemark, Polen, Ungarn, Rumänien). Diese Zerteilung wurde großenteils verursacht durch die - zeitweise extrem imperiale und auch aggressive - Politik des größten deutschsprachigen Staates, Deutschlands, und entgegen gesetzter Maßnahmen der Nachbarländer. Helmut Plessner (1959) hat hinsichtlich dieser Geschichte von einer „verspäteten deutschen Nation“ gesprochen, was zwar als übertrieben kritisiert wurde, aber wesentliche Aspekte andeutet. Der Terminus ließe sich mutatis mutandis auch auf die italienische Nation beziehen. Dass beide Sprachen heute dennoch zu den internationalen Sprachen zählen, ist vor allem der Wirtschaftskraft und der damit zusammenhängenden Attraktivität ihrer Sprachgemeinschaften zuzuschreiben. Beide Sprachen rangieren unter den ersten 10 wirtschaftsstärksten Sprachen der Welt (nach Bruttosozialprodukt ihrer Muttersprachler und Amtssprachgebiete): Deutsch ungefähr auf dem 4. und Italienisch dem 9. Rangplatz - „ungefähr“, weil sich die Plätze von Zeit zu Zeit etwas verschieben (Ammon 2015: 193). Die besondere geographische Lage des deutschen Sprachgebiets hat mich motiviert, den mit dieser Sprache verbundenen Tourismus auf den Aspekt zu beschränken, den die folgende Überschrift anzeigt. 


\section{Deutsch als plurizentrische Sprache}

Die Konzentration auf den hiermit angedeuteten Aspekt erscheint mir auch deshalb berechtigt, weil er bislang in Sprachuntersuchungen zum Tourismus vernachlässigt wurde - abgesehen von gelegentlichen Hinweisen und ausschnitthaften Befassungen, z.B. auch im Beitrag zum vorliegenden Band von Monika Dannerer/Marianne Franz. Deutsch als plurizentrische Sprache ist inzwischen ein theoretisch und empirisch gründlich untersuchtes Thema, woran ich selbst mitgewirkt habe (z.B. Ammon 1995, 2015: 107-405 passim, 2017; Ammon/Bickel/Lenz 2016). Ein klar gegliederter Überblick findet sich im Buch von Birte Kellermeier-Rehbein (2014). Außer Deutsch sind noch eine ganze Reihe anderer Sprachen plurizentrisch, die sich als staatliche Amtssprache über mehrere Staaten erstrecken, z.B. Englisch, Französisch, Spanisch, Portugiesisch, Italienisch, Chinesisch oder Niederländisch (weitere in Clyne 1992).

Der Terminus plurizentrisch bezieht sich allerdings nicht genau auf Staaten, sondern - wie er es ausdrückt - auf Zentren. Damit sind vor allem diejenigen Staaten oder Regionen gemeint, die über spezifische standardsprachliche Varianten der betreffenden Sprache verfügen, also gewissermaßen über eine eigene Standardvarietät. Für die Amtssprache eines Staates gibt es in aller Regel auch spezifische amtliche und damit standardsprachliche Ausdrücke. Meist kommen jedoch weitere standardsprachliche Eigenheiten dazu, vor allem im Wortschatz, oft aber auch auf anderen grammatischen Ebenen (Rängen) wie der Schreibung (Orthographie), Aussprache (Orthophonie), Grammatik und auch Pragmatik (z.B. Grußformen). Als standardsprachlich gelten dabei, grob gesprochen, alle Varianten, die auch in der (größeren) Öffentlichkeit anstandslos gebraucht werden dürfen, also im öffentlichen Sprachgebrauch normal sind (Begriffserläuterung z.B. in Ammon 1995: 73-88, 2017). Ausführliche Beschreibungen für die verschiedenen Zentren der deutschen Sprache finden sich im Variantenwörterbuch des Deutschen (Ammon/Bickel/Lenz 2016) - übrigens dem offenbar bislang einzigen plurizentrischen Wörterbuch überhaupt. Dabei ist der Wörterbuchteil, wegen der besonders vielen Varianten im Wortschatz, am umfangreichsten (S. 1-857); jedoch sind auch die Besonderheiten der normgerechten Schreibung, Aussprache, Grammatik und Pragmatik erfasst, auch in Form übergreifender Regeln (LXIV-LXXVII).

Für dieses Wörterbuch wurden insgesamt 10 Zentren des Deutschen identifiziert, die im Untertitel aufgeführt sind. Ich füge in der folgenden Aufzählung hier hinter jeder Nennung des Zentrums, gewissermaßen als Symbol, ein spezifisches Wort bei: in Kursivschrift, und dahinter, auch als Bedeutungsangabe, eine andere, oft gemeindeutsche Variante (ohne Wiederholung des Artikels bei gleichem Genus): Österreich (die Marille ,Aprikose'), deutschsprachige Schweiz (der Bostitsch ,Tacker'), Deutschland (die Apfelsine ,Orange`), Liechtenstein (der Landesphysikus ,Amtsarzt'), Luxemburg (recyclieren, recyceln'), Ostbelgien (Animation, Veranstaltung'), Südtirol/Alto Adige (der Hydrauliker ,Klempner'), Rumänien (die Bierfabrik ,Bierbrauerei'), Namibia 
(das Bokkie ,die Ziege') und Mennonitensiedlungen, verstreut über den ganzen amerikanischen Kontinent (Grünhaus ,Gewächshaus'). Die 3 zuletzt genannten Zentren sind erst in der 2., völlig neu bearbeiteten Auflage hinzu gekommen und waren in der Erstauflage (2004) noch nicht enthalten.

Nach dem Grad der institutionellen und normativen Absicherung, der Selbstverständlichkeit der Anerkennung als standardsprachlich innerhalb des eigenen Zentrums und der Zahl spezifischer Varianten wurden dreierlei Typen von Zentren der deutschen Sprache unterschieden, nämlich „Voll-“, „Halb-“ und „Viertelzentren“ (Ammon/Bickel/Lenz 2016: XXXIX-LXIII). In den Voll- und Halbzentren ist Deutsch staatliche Amtssprache. Die 3 Vollzentren (Deutschland, Österreich, deutschsprachige Schweiz) haben zudem standardsprachliche Besonderheiten nicht nur im Wortschatz, sondern auf allen sprachlichen Ebenen (Orthographie, Orthophonie, Grammatik und Pragmatik). Außerdem verfügen sie über eine eigene Kodifizierung ihrer Standardvarietät (Deutschland: vor allem die Dudenbände, besonders Rechtschreibung; Österreich: Österreichisches Wörterbuch; die (deutschsprachige) Schweiz: Schweizer Wörterbuch). Die 7 Halb- und Viertelzentren haben nur Besonderheiten im Wortschatz und keine eigenen Nachschlagewerke. In den Viertelzentren, die ebenfalls nur Wortschatzbesonderheiten haben, ist Deutsch auch keine staatliche Amtssprache, sondern nur anerkannte Minderheitensprache. Dort sind die standardsprachlichen Varianten als solche nur gestützt durch die Akzeptanz seitens ihrer Sprachgemeinschaft für den öffentlichen Sprachgebrauch: im Schulunterricht, in schriftlichen Texten auch der Sachliteratur (nicht nur Unterhaltung) und in örtlichen Medien, vor allem Zeitungen.

Die 7 Voll- und Halbzentren bilden das Amtssprachgebiet (oder die Amtssprachregion) der deutschen Sprache. Davon kann man noch das Muttersprachgebiet des Deutschen unterscheiden, wo Deutsch die in der Wohnbevölkerung vorherrschende Muttersprache ist. Das Halbzentrum Luxemburg gehört nicht dazu, weil seine Bevölkerung mit großer Mehrheit sich nicht zu Deutsch, sondern Luxemburgisch (Lëtzebuergesch) als Muttersprache bekennt. Zum Muttersprachgebiet des Deutschen gehören jedoch die Viertelzentren, die aber schwer genau abgrenzbar sind. Dies gilt erst recht für die Wohngebiete sonstiger deutschsprachiger Minderheiten in Europa und der Welt (Ammon 2015: 199-405), die nicht einmal zu den Viertelzentren zählen, weil für sie - jedenfalls bislang - keine standardsprachlichen Besonderheiten der deutschen Sprache nachgewiesen sind. Zur Veranschaulichung eines Ausschnitts der Plurizentrik des Deutschen mögen hier die beiden Karten genügen (weitere Karten und Erläuterungen in Ammon/Bickel/Lenz 2006: XXXIX-LXIII). 


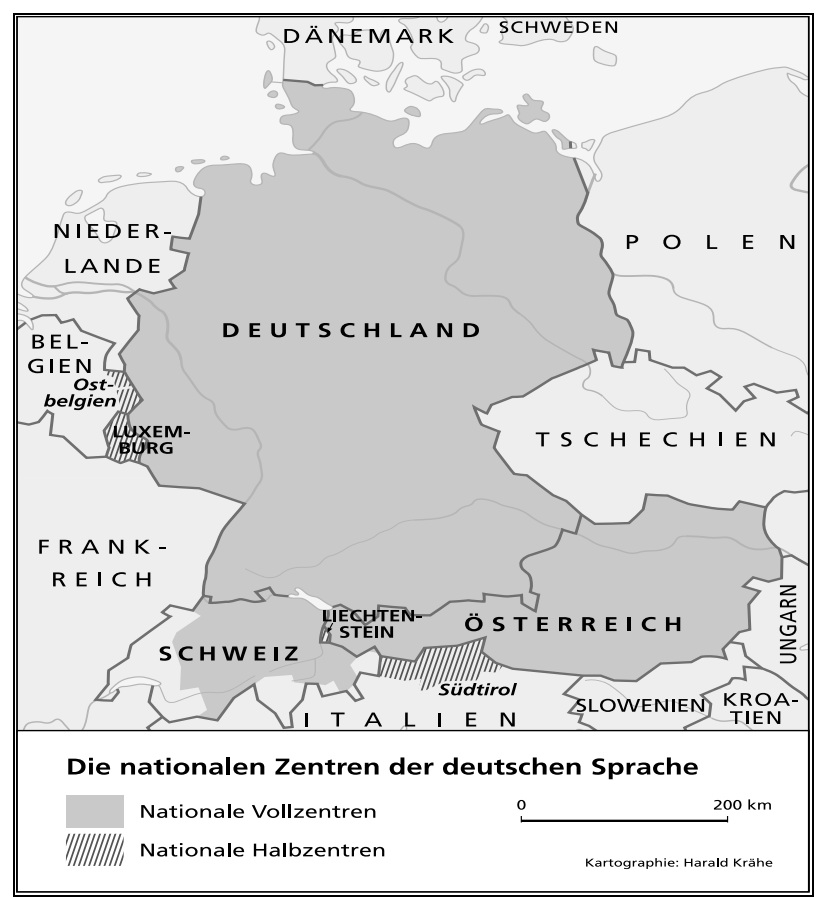

Karte 1: Die nationalen Zentren der deutschen Sprache

\section{Der Intertourismus zwischen den Zentren der deutschen Sprache}

Die Wirtschaftskraft der Zentren der deutschen Sprache ist die - wenngleich prosaisch anmutende - Grundlage für den Tourismus, speziell den Ausreise-Tourismus aus den Zentren oder Ländern der deutschen Sprache. Dies gilt ebenso für den Inter-Tourismus, wie ich ihn hier nennen möchte, zwischen den Zentren. Für diesen Tourismus innerhalb der Plurizentrik des Deutschen ist sehr wahrscheinlich die gemeinsame Sprache ein stimulierender Faktor; er wäre ohne sie weniger intensiv. Allerdings erweist sich der ernsthafte Nachweis oder gar die Ermittlung des Gewichts dieses Faktors bei näherer Betrachtung als schwierige Aufgabe - so schwierig, dass sie, soweit ich sehe, bisher nicht ernsthaft in Angriff genommen wurde und ich mich hier mit spärlichen Hinweisen begnügen muss. 


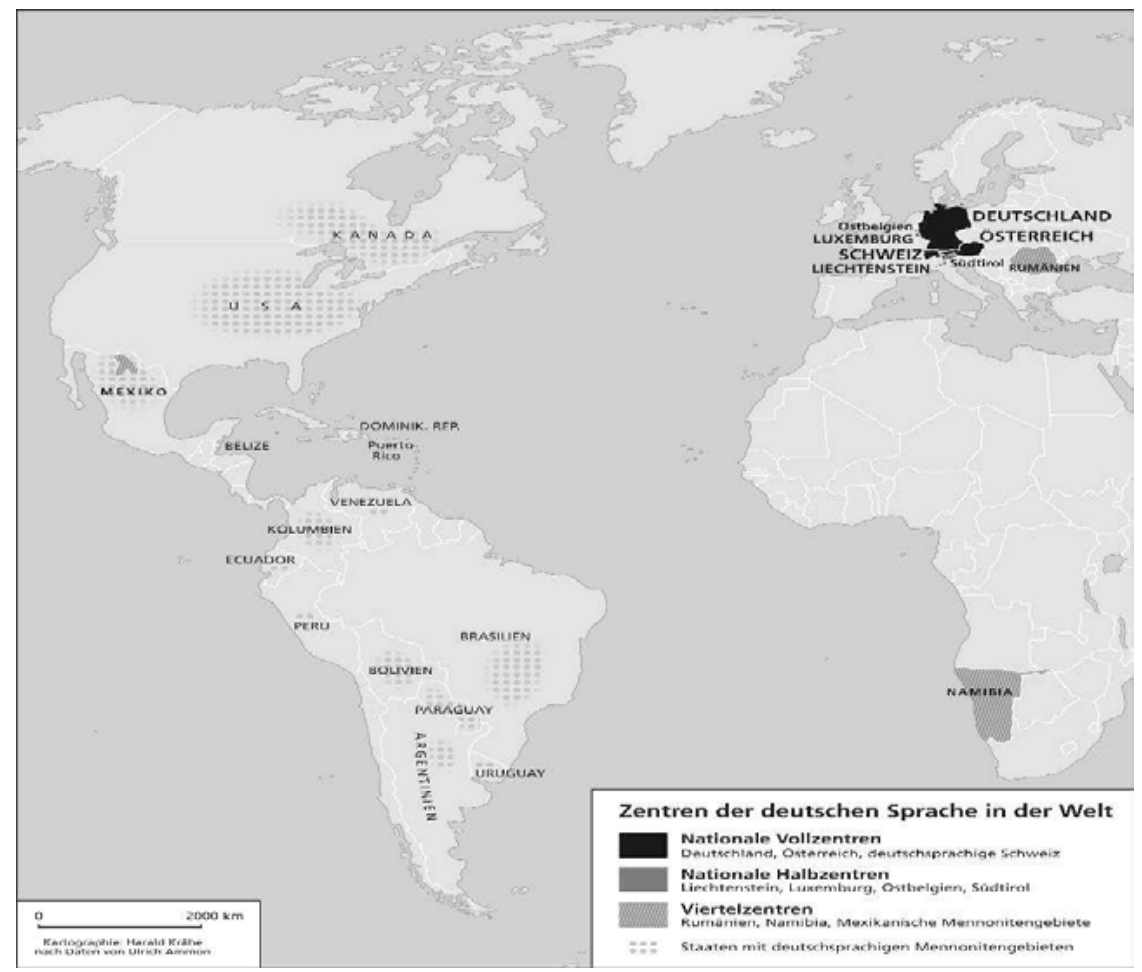

Karte 2: Zentren der deutschen Sprache in der Welt

Wegen des Größenunterschieds zwischen den Zentren der deutschen Sprache beschränke ich mich auf den Ausreisetourismus aus Deutschland in die anderen Zentren. Deutschland alleine hat weit mehr Einwohner als alle anderen Zentren der deutschen Sprache zusammen (ca. 82 Mio. gegenüber in summa ca. 14 Mio.). Daher ist es nicht erstaunlich, dass die Deutschen die größte deutschsprachige Touristengruppe aus den Zentren der deutschen Sprache bilden - wie außerdem sogar eine der größten nach Sprachen klassifizierten Touristengruppen überhaupt (Ammon 2015: 844853). Ich begnüge mich hier fürs Weitere auf kurze Charakterisierungen des aus Deutschland kommenden Einreise-Tourismus in die beiden Vollzentren Österreich und (deutschsprachige) Schweiz, in das Halbzentrum Südtirol/Alto Adige sowie über Europa - in das Viertelzentrum Namibia.

Österreich gehört seit je zu den beliebtesten touristischen Zielländern der Deutschen. In der Zeitspanne 1970-2012 und vermutlich bis heute hat Österreich unter den touristischen Zielländern der Deutschen nie niedriger rangiert als an dritter Stelle aller Länder der Welt, meist auch vor größeren und touristisch ebenfalls attraktiven Ländern wie Türkei oder sogar Frankreich (Ammon 2015: 850, Tab. I.4-2). Verschiedene Einzelstimmen - systematische Untersuchungen zu der Frage scheinen nicht 
vorzuliegen - lassen vermuten, dass die österreichische Tourismusindustrie sich teilweise sprachlich an die deutschen Gäste anpasst und eigene Varianten (Austriazismen) durch solche aus Deutschland (Teutonismen) ersetzt, z. B. auf Speisekarten (Blumenkohl statt Karfiol, Zander statt Fogosch und dergleichen). Umgekehrt genießen jedoch Deutsche sicher die Austriazismen und vergnügen sich bisweilen an Verwechslungen (z.B. Palatschinke fälschlich als ,Schinken' statt richtig ,Pfannekuchen'). Die gelegentlich geäußerten Sorgen österreichischer Sprachkritiker vor der Verdrängung von Austriazismen durch Teutonismen sollten zwar ernst genommen, aber nicht dramatisiert werden.

Die Schweiz, speziell auch die deutschsprachige, gehört ebenfalls zu den bevorzugten Zielländer von Touristen aus Deutschland, allerdings nach Zahl der Besucher hinter Österreich (Ammon 2015: 850, Tab. I-4.2). Die Schweizer Tourismusstatistik meldet für das Jahr 2016: „Die grösste ausländische Nachfrage verbuchten die deutschen Gäste mit 3,7 Millionen Logiernächten“. (Hinweis von Simone Ueberwasser 02.02.2018; https://www.bfs.admin.ch/bfs/de/home/statistiken/kataloge-datenbanken/publikationen.assetdetail.3282044.html). Welche Rolle die Sprachunterschiede innerhalb der Schweiz (Deutsch, Französisch, Italienisch, Rätoromanisch) für die Wahl der Zielorte von Touristen aus Deutschland spielen, ist schwierig zu ermitteln, da andere Faktoren, landschaftliche oder klimatische, mitspielen. Außerdem ist die deutschsprachige Schweiz für Deutsche sprachlich vielleicht weniger zugänglich als andere Zentren der deutschen Sprache, da die Schweizer zum Gebrauch des Dialekts (Schwitzerdütsch - verschiedene Schreibweisen) neigen, der für die meisten Deutschen, außer für manche Süddeutschen, schwer verständlich ist. Allerdings kommen sie den deutschen Gästen in der Regel sprachlich entgegen. Die Besonderheiten des Schweizer Standarddeutschs (Schweizerhochdeutsch), das Deutsche oft auch für Dialekt halten, werden von Deutschen vermutlich oft als Teil des gesuchten besonderen Ambientes geschätzt. Mögliche Missverständnisse werden anekdotisch scherzhaft übertrieben werden - dass deutschen Gästen, die ein „Hörnchen“ (in Deutschland ein Süßgebäck) ordern, dann eine einzelne, gebogene Nudel (schweizerhochdeutsch Hörnli) serviert werde, oder als „Müsli“ eine gekochte Maus (schweizerhochdeutsch Müesli, auch mit fallendem Diphthong gesprochen). Die Sprachschwierigkeiten sind in Wirklichkeit minimal. Allerdings gibt es gelegentliche Aversionen gegen Deutsche, von denen so viele in der Schweiz arbeiten (wegen der höheren Löhne) und mit den einheimischen Schweizern um attraktive Stellen konkurrieren (schweizerhochdeutsch konkurrenzieren).

Für das Halbzentrum Südtirol/Alto Adige sind die Touristen aus Deutschland ebenfalls die größte Einreise-Gruppe (Ammon 2015: 247). Die italienische Provinz ist amtlich zweisprachig: Deutsch und Italienisch, und die Zweisprachigkeit wird vor allem gestützt durch getrennte Schulen, in denen entweder Deutsch Unterrichtssprache ist und Italienisch Schulfach oder umgekehrt. Zwar sind die Deutschsprachigen in der Überzahl (ca. 62\%), aber die Italienischsprachigen (ca. 23\%) haben die - zu- 
mindest psychologisch wirksame - Rückendeckung des Gesamtstaates mit der alleinigen Amtssprache Italienisch. Die kleine Gruppe der Ladinischsprachigen (ca. 4\%) steht wegen der Sprachähnlichkeit den Italienischsprachigen, aber wegen des Minderheitenstatus bezüglich des Gesamtstaates den Deutschsprachigen näher (Zahlen nach https://de.wikipedia.org/wiki/S\%C3\%BCdtirol; abgerufen 02.02.2018). Der Tourismus ist für die Provinz einer der wichtigsten Wirtschaftszweige, und die Touristen aus Deutschland sind die mit Abstand größte Gruppe.

Sie trägt vermutlich maßgeblich zum Erhalt der deutschen Sprache bei und auch dazu, dass jedenfalls im Tourismus eher Entlehnungen aus dem Standarddeutsch Deutschlands als Österreichs im Gebrauch sind (Lanthaler 2012: 77), trotz größerer geographischer und sprachlicher Nähe (gemeinsamer Dialekt Südbairisch) sowie politischer Zugehörigkeit zu Österreich bis 1919. Möglicherweise trägt der Tourismus aus Deutschland auch dazu bei, dass ins Südtiroler Standarddeutsch heute weniger neue Entlehnungen aus dem Italienischen kommen als früher - wenn auch Letztere immer noch charakteristisch sind (Aranciata ,Orangenlimonade', Bar ,Café', ajournieren ,auf den neuesten Stand bringen', Marende ,Zwischenmahlzeit', Carabiniere ,(Militär)Polizist' usw.).

Das Viertelzentrum Namibia hat seine deutschsprachige Minderheit aus der kurzen Zeit, als das Gebiet die Kolonie Deutsch Südwestafrika, die einzige Siedlungskolonie, des Deutschen Reiches war (1874 - 1915, Besetzung durch Südafrika, oder 1920, Mandat Südafrikas). Die von dort überkommenen und später teilweise durch Zuzug verstärkten Deutschsprachigen bilden zwar eine kleine Minderheit (ca. 25.000), die aber wirtschaftlich und auch sprachlich auffällig präsent ist (Zappen-Thomson im Druck; Ammon 2015: 359-369). Daher kann man im dortigen Urlaub sich ohne nennenswerte Schwierigkeiten ganz auf Deutsch verständigen. Damit wirbt auch Namibias Tourismusindustrie, und mit Erfolg. Aus den deutschsprachigen Ländern kommen seit Jahren regelmäßig die meisten Einreisetouristen. Für das Jahr 2017 wurden die folgenden Zahlen für die größten Herkunftsländer von Touristen festgestellt: Deutschland + Österreich + Schweiz 190.684 (27,1\%), Südafrika 80.901 (11,5 \%), Frankreich 28,963 (4,1\%), USA + Kanada 26.294 (3,7 \%), UK + Irland 24.034 (3,4 \%), Italien 21.053 (3,0 \%) („Tourismusjahr ist wieder top“; Allgemeine Zeitung, Windhoek, 25.01.2018). Marianne Zappen-Thomson, Germanistikprofessorin an der Universität der namibischen Hauptstadt Windhoek, hat mir diese Daten übermittelt und dazu mitgeteilt, dass „der Anstieg der DaF-SchülerInnen [in Namibia! U.A.] sehr erfreulich [sei]. Ein Grund dafür liegt gewiss auch an den größeren Chancen auf dem Arbeitsmarkt im Tourismussektor.“ (DaF = Deutsch als Fremdsprache; E-Mail 02.02.2018) Es erscheint kaum übertrieben, dass die deutsche Sprache in Namibia erheblichen Teils durch den Tourismus aus den deutschsprachigen Ländern gestützt wird. In fast allen Restaurants oder Quartieren findet sich deutschsprachiges Personal, und die deutsche Sprache ist in den Sprachlandschaften vieler Ortschaften präsent. 


\section{Literaturverzeichnis}

Ammon, Ulrich (1995): Die deutsche Sprache in Deutschland, Österreich und der Schweiz. Das Problem der nationalen Varietäten. Berlin/New York: Walter de Gruyter.

Ammon, Ulrich (2015): Die Stellung der deutschen Sprache in der Welt. Berlin/München/Boston: Walter de Gruyter.

Ammon, Ulrich (2017): Grundbegriffe für plurizentrische Sprachen und ihre Operationalisierung mit Bezug auf die deutsche Sprache und das „Variantenwörterbuch des Deutschen“. In: Zeitschrift für deutsche Philologie 136. Band 2017 - Sonderheft: 21, 5-21.

Ammon, Ulrich/Bickel, Hans/Lenz, Alexandra [1. Aufl. 2004] (2016): Variantenwörterbuch des Deutschen. Die Standardsprache in Österreich, der Schweiz, Deutschland, Liechtenstein, Luxemburg, Ostbelgien und Südtirol sowie Rumänien, Namibia und Mennonitensiedlungen. 2., neu bearb. Aufl. Berlin/Boston: Walter de Gruyter.

Kellermeier-Rehbein, Birte (2014): Plurizentrik. Einführung in die nationalen Varietäten des Deutschen. Berlin: Erich Schmidt.

Clyne, Michael (ed.) (1992): Pluricentric Languages. Differing Norms in Different Nations. Berlin/New York: Mouton de Gruyter.

Duden. Die deutsche Rechtschreibung (2017): 27. Aufl. Berlin/Mannheim: Dudenverlag.

Lanthaler, Franz [1996] (2012): Varietäten des Deutschen in Südtirol. In: Drubl, Hans/Sitta, Horst (eds.): Texte zu Sprache und Schule in Südtirol. Meran: Edizioni alphabeta, 69-91.

Meyer, Kurt (2006): Schweizer Wörterbuch. So sagen wir in der Schweiz. Mit einem Beitrag von Hans Bickel. Frauenfeld/Stuttgart/Wien: Huber.

Plessner, Helmuth (1959): Die verspätete Nation. Über die politische Verführbarkeit bürgerlichen Geistes. Stuttgart: W. Kohlhammer.

Österreichisches Wörterbuch (2016) (red.): Pabst, Christiane M./Fussy, Herbert/Steiner, Ulrike. 43. Aufl. Wien: öbv.

Zappen-Thomson, Marianne (im Druck): Stützung des Spracherhalts bei deutschsprachigen Minderheiten: Namibia. In: Ammon, Ulrich/Schmidt, Gabriele (eds.): Förderung der deutschen Sprache weltweit. Vorschläge, Ansätze und Konzepte. Berlin/München/Boston: Walter de Gruyter. 


\section{DuEPublico}

Duisburg-Essen Publications online
UNIVERSITÄT

$D_{E} U_{S} S_{S} S_{N}$ U R G

offen im Denken

$\mathbf{U b} \mid \begin{aligned} & \text { universitäts } \\ & \text { bibliothek }\end{aligned}$

Aus: Sociolinguistica (2018), 32(1), S. 69 ff.

Dieser Text wird über DuEPublico, dem Dokumenten- und Publikationsserver der Universität Duisburg-Essen, zur Verfügung gestellt. Die hier veröffentlichte Version der EPublikation kann von einer eventuell ebenfalls veröffentlichten Verlagsversion abweichen.

DOI: $\quad 10.1515 /$ soci-2018-0007

URN: urn:nbn:de:hbz:464-20200721-081958-8

\section{Alle Rechte vorbehalten.}

Dieser Beitrag ist mit Zustimmung des Rechteinhabers aufgrund einer (DFG-geförderten) Allianz- bzw. Nationallizenz frei zugänglich. 